UT-STPD-4/98

BA-98-11

\title{
R-Symmetry in the Minimal Supersymmetric Standard Model and Beyond with Several Consequences
}

\author{
G. Lazarides ${ }^{(1) *}$ and Q. Shafi ${ }^{(2) \dagger}$ \\ (1) Physics Division, School of Technology, Aristotle University of Thessaloniki, \\ Thessaloniki GR 540 06, Greece. \\ ${ }^{(2)}$ Bartol Research Institute, University of Delaware, \\ Newark, DE 19716, USA
}

\begin{abstract}
The supersymmetric sector of minimal supersymmetric standard model (MSSM) possesses a $U(1)$ R-symmetry which contains $Z_{2}$ matter parity. Non-zero neutrino masses, consistent with a 'redefined' R-symmetry, are possible through the see-saw mechanism and/or a pair of superheavy (mass M) $S U(2)_{L}$ triplets with vev $\sim M_{W}^{2} / M$. If this R-symmetry is respected by the higher order terms, then baryon number conservation follows as an immediate consequence. In the presence of right handed neutrinos, the observed baryon asymmetry of the universe arises via leptogenesis. An interplay of R- and Peccei-Quinn symmetry simultaneously resolves the strong $\mathrm{CP}$ and $\mu$ problems.
\end{abstract}

\footnotetext{
*lazaride@eng.auth.gr

${ }^{\dagger}$ shafi@bartol.udel.edu
} 
Although quite compelling, the minimal supersymmetric standard model (MSSM) fails to address a number of important challenges. For instance, to explain the apparent stability of the proton, it must be assumed that the dimensionless coefficients accompanying dimension five operators are of order $10^{-8}$ or less. The strong CP and $\mu$ problems loom large in the background, and the observed baryon asymmetry, it appears, cannot be explained within the MSSM framework. Last, but by no means least, there is increasing evidence for non-zero neutrino masses from a variety of experiments.

In a recent paper [1], we offered one approach for resolving many of the above problems. It relied on extending the gauge symmetry to $S U(2)_{L} \times S U(2)_{R} \times U(1)_{B-L}$, with a global $U(1)$ R-symmetry playing an essential role. The magnitude of the supersymmetric $\mu$ term of MSSM was directly related to the gravitino mass $m_{3 / 2}(\sim 1 \mathrm{TeV})$ which, in turn, arises from the hidden sector a la supergravity. The left-right symmetry ensures the presence of right handed neutrino superfields and consequently non-zero neutrino masses, while the R-symmetry implies an accidental global $U(1)_{B}$ symmetry which explains why the proton is so stable. Note that the R-symmetry is spontaneously and perhaps even explicitly broken by the hidden sector. The soft (quadratic and trilinear) supersymmetry breaking terms in the visible sector are expected to explicitly break the R-symmetry.

In this paper, we wish to provide a resolution of the problems listed above without departing from the $S U(3)_{c} \times S U(2)_{L} \times U(1)_{Y}$ framework of MSSM. We observe that the MSSM superpotential $W$ possesses a global $U(1)$ R-symmetry [2] in which $Z_{2}$ matter parity is embedded. We show how neutrino masses can be incorporated while preserving a (redefined) R-symmetry. When extended to higher orders, this symmetry ensures the appearance of global $U(1)_{B}$, thereby guaranteeing proton stability. In the case where right handed neutrinos are included, the observed baryon asymmetry of the universe can arise, as we will see, via leptogenesis. The approach followed here also provides the framework for an elegant resolution of the strong CP and $\mu$ problems of MSSM, with the R-symmetry once again playing an essential role.

The MSSM superpotential $W$ contains the following renormalizable terms (we will not distinguish between the generations in this paper):

$$
H^{(1)} Q U^{c}, H^{(2)} Q D^{c}, H^{(2)} L E^{c}, H^{(1)} H^{(2)} .
$$


Here $H^{(1)}, H^{(2)}$ are the two higgs superfields, $Q$ denotes the $S U(2)_{L}$ doublet quark superfields, $U^{c}$ and $D^{c}$ are the $S U(2)_{L}$ singlet quark superfields, while $L\left(E^{c}\right)$ stands for the $S U(2)_{L}$ doublet (singlet) lepton superfields. A $Z_{2}$ matter parity $\left(Z_{2}^{m p}\right)$ under which only the 'matter' superfields change sign ensures the absence of terms such as $Q D^{c} L$ and $U^{c} D^{c} D^{c}$ which lead to rapid proton decay.

The superpotential in Eq.(1]) possesses three global symmetries, namely, $U(1)_{B}, U(1)_{L}$ and $U(1)_{R}$. (Except for sphaleron effects in baryogenesis, we will ignore the 'tiny' nonperturbative violation of $B$ and $L$ by the $S U(2)_{L}$ instantons.) The 'global' charges of the various superfields are as follows:

$$
\begin{gathered}
B: H^{(1)}(0), H^{(2)}(0), Q(1 / 3), U^{c}(-1 / 3), D^{c}(-1 / 3), L(0), E^{c}(0) ; \\
L: H^{(1)}(0), H^{(2)}(0), Q(0), U^{c}(0), D^{c}(0), L(1), E^{c}(-1) ; \\
R: H^{(1)}(1), H^{(2)}(1), Q(1 / 2), U^{c}(1 / 2), D^{c}(1 / 2), L(1 / 2), E^{c}(1 / 2) .
\end{gathered}
$$

We have normalized the $R$ charges such that $W$ carries two units.

The introduction of the right handed neutrino superfields, $\nu^{c}$, gives rise, consistent with $Z_{2}^{m p}$, to two additional renormalizable superpotential couplings

$$
H^{(1)} L \nu^{c}, M^{R} \nu^{c} \nu^{c}
$$

where $M^{R}$ is the Majorana mass matrix of the superheavy right handed neutrinos. The first term in Eq.(B) fixes the quantum numbers of the right handed neutrinos, namely, $B\left(\nu^{c}\right)=0, L\left(\nu^{c}\right)=-1, R\left(\nu^{c}\right)=1 / 2$. The second term violates both $U(1)_{L}$ and $U(1)_{R}$, but the combination

$$
R^{\prime}=R-\frac{1}{2} L
$$

is now the new R-symmetry of the superpotential. In addition, the $Z_{2}^{l p}$ (lepton parity) subgroup of $U(1)_{L}$, under which only the lepton superfields $L, E^{c}$ change sign, remains unbroken. Consequently, the global symmetries of the renormalizable superpotential containing all the couplings in Eqs.(1) and (3) are $U(1)_{R^{\prime}}, U(1)_{B}$ and $Z_{2}^{l p}$. With the 
couplings in Eq.(3), the observed neutrinos acquire masses via the well-known see-saw mechanism [3].

It is interesting to note that both $U(1)_{B}$ and lepton parity are automatically implied by $U(1)_{R^{\prime}}$. Moreover, this remains true even if non-renormalizable terms are included in the superpotential. Indeed, by extending the $U(1)_{R^{\prime}}$ symmetry to higher order terms, we will first show that $U(1)_{B}$ follows as a consequence. To see this, note that $U(1)_{R^{\prime}}$ contains $Z_{2}^{b p}$ (baryon parity) under which only the color triplet, antitriplet $(3, \overline{3})$ superfields change sign. This means that superpotential couplings containing, in addition to color singlet and $(3 \cdot \overline{3})^{m}(\mathrm{~m} \geq 0)$ factors, the $U(1)_{B}$ violating combinations $(3 \cdot 3 \cdot 3)^{n}$ or $(\overline{3} \cdot \overline{3} \cdot \overline{3})^{n}$ with $n=$ odd $\geq 1$ are not allowed. Similarly, analogous couplings but with $n=$ even $\geq 2$ are also not allowed since their $R^{\prime}$ charge exceeds two units and cannot be compensated. In particular, the troublesome dimension five operators $Q Q Q L$ and $U^{c} U^{c} D^{c} E^{c}$ are eliminated.

One can next show that $U(1)_{R^{\prime}}$ implies $Z_{2}^{l p}$ (lepton parity) to all orders. Because of $U(1)_{B}$, the quark superfields must appear in 'blocks' $Q U^{c}(1)$ and $Q D^{c}(1)$, where the parenthesis indicates the $R^{\prime}$ charge. The other non-leptonic 'blocks' are $H^{(1)}(1)$ and $H^{(2)}(1)$. The leptonic superfields are $L(0), E^{c}(1), \nu^{c}(1)$. To violate lepton parity, we need an odd number of lepton superfields. Therefore, we should consider: i) odd number of $L$ 's together, by $U(1)_{R^{\prime}}$ symmetry, with two non-leptonic blocks belonging to the four types described above; ii) even number of $L$ 's and a single $E^{c}$ or $\nu^{c}$, together with one non-leptonic block; iii) odd number of $L$ 's with two out of the $E^{c}$ 's and $\nu^{c}$ 's. In all three cases, one ends up with an odd number of $S U(2)_{L}$ doublets which is not gauge invariant.

In summary, both lepton parity and $U(1)_{B}$ are present in the scheme to all orders as mere consequences of the $U(1)_{R^{\prime}}$ symmetry and remain exact, although $U(1)_{R^{\prime}}$ is explicitly broken to its maximal non-R-subgroup $Z_{4}^{\prime}$ (which includes $Z_{2}^{b p}$ ) by the supersymmetry breaking terms in the visible sector.

We now present an alternative scheme for introducing non-zero neutrino masses in MSSM . This scheme is, actually, quite familiar « from Grand Unified Theories (GUTs), and was recently considered within the non-supersymmetric standard model framework in Ref. [5]. Introduce, in MSSM, an $S U(2)_{L}$ triplet pair $T$, $\bar{T}$, with hypercharges $+1,-1$ 
respectively. Consider the renormalizable superpotential couplings

$$
T L L, \bar{T} H^{(1)} H^{(1)}, M T \bar{T},
$$

such that $B(T)=B(\bar{T})=0, L(T)=-2, L(\bar{T})=0, R(T)=1, R(\bar{T})=0$, from the first two couplings, and $M$ is some superheavy scale (taken real and positive by suitable phase redefinitions of the superfields $T, \bar{T}$ ). The supersymmetric mass term in Eq.(5) breaks $U(1)_{R}$ and $U(1)_{L}$ but, in analogy with the previous discussion involving the $\nu^{c}$ superfields, the superpotential defined by the terms in Eqs.(1) and (5) possesses a redefined R-symmetry generated by

$$
R^{\prime \prime}=R-\frac{1}{2} L
$$

The $R^{\prime \prime}$ charges of the various superfields are:

$$
H^{(1)}(1), H^{(2)}(1), Q(1 / 2), U^{c}(1 / 2), D^{c}(1 / 2), L(0), E^{c}(1), T(2), \bar{T}(0) .
$$

Both $U(1)_{B}$ and lepton parity remain unbroken in this case too. Finally, as with the $U(1)_{R^{\prime}}$ symmetry, one can readily show that $U(1)_{R^{\prime \prime}}$ implies conservation of $B$ and $Z_{2}^{l p}$ to all orders despite its explicit breaking to its maximal non-R-subgroup $Z_{4}^{\prime \prime}$ (including $Z_{2}^{b p}$ ) by the supersymmetry breaking terms in the visible sector.

It is readily checked that the scalar component of $T$ acquires a non-zero vacuum expectation value $(\mathrm{vev}) \sim M_{W}^{2} / M\left(\ll M_{W}\right)$, with the electroweak breaking playing an essential role in the generation of this vev. This is due to the fact that the two last terms in Eq.(5), after electroweak breaking, give rise to a term linear with respect to $T$ in the scalar potential of the theory. The vev of $T$ leaves $U(1)_{B}, Z_{2}^{l p}$ and the $Z_{4}^{\prime \prime}$ subgroup of $U(1)_{R^{\prime \prime}}$ unbroken and generates non-zero neutrino masses. Note that $T, \bar{T}$ are superheavy fields, so that the low energy spectrum is given by the MSSM.

It should be clear that the coexistence of all the superpotential couplings in Eqs.(1), (5) and (5) provides us with a scheme where the light neutrino masses acquire contributions from the see-saw mechanism as well as the triplet vev. It is important to note that, in this 'combined' case, $W$ possesses a $U(1)$ R-symmetry, $U(1)_{\hat{R}}$, which coincides with $U(1)_{R^{\prime}}$ or $U(1)_{R^{\prime \prime}}$ when restricted to the superfields where these symmetries are defined. (Note that $U(1)_{R^{\prime}}$ and $U(1)_{R^{\prime \prime}}$ become identical when restricted to the MSSM 
superfields.) This R-symmetry, just as in the previous cases, implies $U(1)_{B}$ and $Z_{2}^{l p}$ to all orders. It is, finally, interesting to notice that baryon number and lepton parity conservation is a consequence of the 'redefined' R-symmetries in Eqs.(田), (6) or $U(1)_{\hat{R}}$, in the 'combined' case, and not of the original $U(1)_{R}$ which allows couplings like $Q Q Q L$ and $U^{c} U^{c} D^{c} E^{c}$.

The two mechanisms considered above for generating masses for the neutrinos have an additional far reaching consequence. This has to do with the generation of the observed baryon asymmetry in the universe. The basic idea is to generate an initial lepton asymmetry which is partially transformed through the non-perturbative electroweak sphaleron effects, that 'actively' violate $B+L$ at energies above $M_{W}$, to the observed baryon asymmetry. Actually, this is the only way to generate baryons in the present scheme, since baryon number is otherwise exactly conserved. This mechanism has been well documented [6] when the lepton asymmetry is created by a decaying massive Majorana neutrino (say from the $\nu^{c}$ superfields) and exploits the couplings given in Eq.(3). If the $T, \bar{T}$ superfields with the couplings given in Eq.(5) are also present, then additional diagrams must be considered.

The complete set of double-cut diagrams for leptogenesis from a decaying fermionic $\nu^{c}$, which is the relevant case for inflationary models where the inflaton predominantly decays to a fermionic right handed neutrino, is displayed in Fig. 1. The resulting lepton asymmetry, in this case, can be estimated [7] to be

$$
\frac{n_{L}}{s} \approx \frac{3}{16 \pi} \frac{T_{r}}{m_{i n f l}} M_{i}^{R} \frac{\operatorname{Im}\left(M^{D} m^{\dagger} \tilde{M}^{D}\right)_{i i}}{\left|\left\langle H^{(1)}\right\rangle\right|^{2}\left(M^{D} M^{D \dagger}\right)_{i i}} .
$$

Here $T_{r}$ is the 'reheat' temperature, $m_{i n f l}$ the inflaton mass, $M^{D}$ the neutrino 'Dirac' mass matrix in the basis where the Majorana mass matrix of right handed neutrinos, $M^{R}$, is diagonal with positive entries, and $M_{i}^{R}$ is the mass of the decaying $\nu_{i}^{c}$. Also

$$
m \approx-\alpha t \frac{\left\langle H^{(1)}\right\rangle^{2}}{M}-\tilde{M}^{D} \frac{1}{M^{R}} M^{D}
$$

is the light neutrino mass matrix in the same basis, with $t$ (a complex symmetric matrix) and $\alpha$ being the coefficients of the first and second terms in Eq.(5) respectively. It should be noted that this estimate holds provided that $M_{i}^{R}$ is much smaller than the mass of the other $\nu^{c}$ 's and the mass $M$ of the triplets . Eq.(8) gives [7] the bound 


$$
\left|\frac{n_{L}}{s}\right| \lesssim \frac{3}{16 \pi} \frac{T_{r}}{m_{\text {infl }}} \frac{M_{i}^{R} m_{\nu_{\tau}}}{\left|\left\langle H^{(1)}\right\rangle\right|^{2}},
$$

which, for $T_{r} \approx 10^{9} \mathrm{GeV}$ (consistent with the gravitino constraint), $m_{\text {infl }} \approx 3 \times 10^{13} \mathrm{GeV}$, $M_{i}^{R} \approx 10^{10} \mathrm{GeV}$ (see Ref. [7]), $\left|\left\langle H^{(1)}\right\rangle\right| \approx 174 \mathrm{GeV}$, and $m_{\nu_{\tau}} \approx 5 \mathrm{eV}$ (providing the hot dark matter of the universe), gives $\left|n_{L} / s\right| \lesssim 3 \times 10^{-9}$. This is large enough to account for the observed baryon asymmetry of the universe. It is important though to ensure that the lepton asymmetry is not erased by lepton number violating $2 \rightarrow 2$ scatterings at all temperatures between $T_{r}$ and $100 \mathrm{GeV}$. This requirement gives $\left[8 m_{\nu_{\tau}} \lesssim 10 \mathrm{eV}\right.$.

We pointed out that, in non-inflationary (and perhaps some inflationary) models, leptogenesis from the decay of bosonic $\nu^{c}$ 's as well as bosonic and fermionic $T, \bar{T}$ 's may be present too. Most of the relevant double-cut diagrams can be obtained from the ones in Fig. 1 by breaking up the $\nu^{c}, T, \bar{T}$ internal lines and joining the external $\nu^{c}$ lines. The only extra diagram (not obtainable this way) is a diagram of the same type with bosonic $\nu^{c}$ external lines and a fermionic $T, \bar{T}$ internal line. The important observation is that diagrams of the type in Fig.11 with no $\nu^{c}$ internal or external lines cannot be constructed. Thus, efficient leptogenesis can take place only in the presence of $\nu^{c}$ 's.

We have seen how $U(1)_{B}$ arises as a consequence of requiring the superpotential $W$ (including higher order terms) to respect a $U(1)$ R-symmetry. Among other things, this explains why the proton is so stable. However, the learned reader may be concerned that requiring the non-renormalizable terms in the superpotential to respect a continuous Rsymmetry may not be a reasonable thing to do. Indeed, one may wonder if continuous global symmetries such as $U(1)_{\hat{R}}$ or the Peccei-Quinn [9] $\left(U(1)_{P Q}\right)$ symmetry, rather than being imposed, can arise in some more 'natural' manner. One way how this may occur was pointed out in Ref. [10]. Here, discrete (including R-) symmetries that typically arise after compactification could effectively behave as if they are continuous. Furthermore, such 'continuous' symmetries can be very useful in resolving problems other than the one of proton stability. To see this, let us now address the strong CP and $\mu$ problems of MSSM. It has been noted by earlier authors [11] that a continuous $U(1)$ R-symmetry can be relevant for the solution of the $\mu$ problem. By invoking $U(1)_{P Q}$ and combining it with the $U(1)$ R-symmetry above, we will provide a resolution of both the strong $\mathrm{CP}$ and $\mu$ problems, with the $U(1)$ R-symmetry playing an essential role in controlling the 
structure of the terms that are permitted at the non-renormalizable level.

It has been recognized for some time that, within the supergravity extension of MSSM, the existence of D- and F-flat directions in field space can generate an intermediate scale $M_{I}$ which, in the simplest case, is given by

$$
M_{I} \sim \sqrt{m_{3 / 2} M_{P}} \sim 10^{11}-10^{12} \mathrm{GeV}
$$

where $m_{3 / 2} \sim 1 \mathrm{TeV}$ is the supersymmetry breaking scale and $M_{P}=1.22 \times 10^{19} \mathrm{GeV}$ is the Planck mass. It seems 'natural' to try and identify $M_{I}$ with the symmetry breaking scale $f_{a}$ of $U(1)_{P Q}$, such that $\mu \sim m_{3 / 2} \sim f_{a}^{2} / M_{P}$ [12]. We will now see how this idea, which simultaneously resolves the strong $\mathrm{CP}$ and $\mu$ problems, can be elegantly realized in the presence of the $U(1)$ R-symmetry. Note that the resolution of the $\mu$ problem forces us to consider non-renormalizable terms.

We supplement the MSSM spectrum with a pair of superfields $N, \bar{N}$ whose vevs will break $U(1)_{P Q}$ at an intermediate scale. $W$ contains [13] the following terms:

$$
H^{(1)} Q U^{c}, H^{(2)} Q D^{c}, H^{(2)} L E^{c}, N^{2} H^{(1)} H^{(2)}, N^{2} \bar{N}^{2}
$$

The global symmetries of this superpotential are $U(1)_{B}, U(1)_{L}$ (with the new superfields $N, \bar{N}$ being neutral under both), an anomalous Peccei-Quinn symmetry $U(1)_{P Q}$, and a non-anomalous R-symmetry $U(1)_{\tilde{R}}$. The $P Q$ and $\tilde{R}$ charges are as follows:

$$
\begin{gathered}
P Q: H^{(1)}(1), H^{(2)}(1), Q(-1 / 2), U^{c}(-1 / 2), D^{c}(-1 / 2), \\
L(-1 / 2), E^{c}(-1 / 2), N(-1), \bar{N}(1), \\
\tilde{R}: H^{(1)}(0), H^{(2)}(0), Q(1), U^{c}(1), D^{c}(1), L(1), E^{c}(1), N(1), \bar{N}(0) .
\end{gathered}
$$

Note that the quartic terms in Eq.(12) carry a coefficient proportional to $M_{P}^{-1}$ which has been left out. The R-symmetry ensures that undesirable terms such as $N \bar{N}$, which otherwise spoil the flat direction in the supersymmetric limit, are absent from Eq.(12).

After taking the supersymmetry breaking terms into account, one finds 13 that, for suitable choice of parameters, a solution with

$$
|\langle N\rangle|=|\langle\bar{N}\rangle| \sim \sqrt{m_{3 / 2} M_{P}}
$$


is preferred over the one with $\langle N\rangle=\langle\bar{N}\rangle=0$. To see this, let us consider the relevant part of the scalar potential:

$$
V=\left(m_{3 / 2}^{2}+\lambda^{2}\left|\frac{N \bar{N}}{M_{P}}\right|^{2}\right)\left(|N|^{2}+|\bar{N}|^{2}\right)+\left(A m_{3 / 2} \lambda \frac{N^{2} \bar{N}^{2}}{2 M_{P}}+h . c\right),
$$

where $\lambda /\left(2 M_{P}\right)$ is the coefficient of the last superpotential term in Eq.(12) and $A$ the dimensionless coefficient of the corresponding soft supersymmetry breaking term $(\lambda$ is taken real and positive by appropriately redefining the phases of $N, \bar{N})$. This potential can be rewritten as

$$
V=\left(m_{3 / 2}^{2}+\lambda^{2}\left|\frac{N \bar{N}}{M_{P}}\right|^{2}\right)\left[(|N|-|\bar{N}|)^{2}+2|N||\bar{N}|\right]+|A| m_{3 / 2} \lambda \frac{|N \bar{N}|^{2}}{M_{P}} \cos (\epsilon+2 \theta+2 \bar{\theta}),
$$

where $\epsilon, \theta, \bar{\theta}$ are the phases of $A, N, \bar{N}$ respectively. Minimization of $V$ then requires $|N|=|\bar{N}|, \epsilon+2 \theta+2 \bar{\theta}=\pi$ and $V$ takes the form

$$
V=2|N|^{2} m_{3 / 2}^{2}\left(\lambda^{2} \frac{|N|^{4}}{m_{3 / 2}^{2} M_{P}^{2}}-|A| \lambda \frac{|N|^{2}}{2 m_{3 / 2} M_{P}}+1\right) .
$$

It is now obvious that, for $|A|>4$, the absolute minimum of the potential is at

$$
|\langle N\rangle|=|\langle\bar{N}\rangle|=\left(m_{3 / 2} M_{P}\right)^{\frac{1}{2}}\left(\frac{|A|+\left(|A|^{2}-12\right)^{\frac{1}{2}}}{6 \lambda}\right)^{\frac{1}{2}} \sim\left(m_{3 / 2} M_{P}\right)^{\frac{1}{2}} .
$$

Note that the $\langle N\rangle,\langle\bar{N}\rangle$ vevs together break $U(1)_{\tilde{R}} \times U(1)_{P Q}$ down to $Z_{2}^{m p}$. Substitution of these vevs in Eq.(12) shows that the $\mu$ parameter of MSSM is of order $m_{3 / 2}$ as desired.

This discussion is readily extended to include either massive right handed neutrino superfields $\nu^{c}$ or the $S U(2)_{L}$ triplet higgs superfields $T, \bar{T}$. In the $\nu^{c}$ case, the new terms in the superpotential $W$ are $H^{(1)} L \nu^{c}$ and $N \nu^{c} \nu^{c}$. The first term yields $B\left(\nu^{c}\right)=0$, $L\left(\nu^{c}\right)=-1, P Q\left(\nu^{c}\right)=-1 / 2, \tilde{R}\left(\nu^{c}\right)=1$. The second term leaves $U(1)_{B}$ unbroken but breaks $U(1)_{L}, U(1)_{P Q}$ and $U(1)_{\tilde{R}}$ to a 'redefined' Peccei-Quinn symmetry $U(1)_{P Q^{\prime}}$ and a 'redefined' R-symmetry $U(1)_{\tilde{R}^{\prime}}$ with $P Q^{\prime}=P Q-L$ and $\tilde{R}^{\prime}=\tilde{R}+P Q-(1 / 2) L$. Thus, the strong CP problem can be resolved. It should be noted that $U(1)_{\tilde{R}^{\prime}}$ coincides with $U(1)_{R^{\prime}}$ in Eq.(4) when restricted to the superfields where $U(1)_{R^{\prime}}$ is defined. Moreover, 
just as $U(1)_{R^{\prime}}$, the R-symmetry $U(1)_{\tilde{R}^{\prime}}$ contains $Z_{2}$ baryon parity as a subgroup and implies $U(1)_{B}$ to all orders. $Z_{2}^{m p}$ is contained in $U(1)_{P Q^{\prime}}$ and, thus, $Z_{2}^{l p}$ is also present but not as an automatic consequence of $U(1)_{\tilde{R}^{\prime}}$ in this case.

A similar discussion applies if the triplets $T, \bar{T}$ are introduced in the scheme of Eq.(12). The new superpotential terms, in this case, are $T L L, \bar{T} H^{(1)} H^{(1)}$ and $N T \bar{T}$. The first two couplings give $B(T)=B(\bar{T})=0, L(T)=-2, L(\bar{T})=0, P Q(T)=1$, $P Q(\bar{T})=-2, \tilde{R}(T)=0$ and $\tilde{R}(\bar{T})=2$. The last coupling leaves unbroken the symmetries $U(1)_{B}, U(1)_{P Q^{\prime \prime}}$ and $U(1)_{\tilde{R}^{\prime \prime}}$ with $P Q^{\prime \prime}=P Q-L$ and $\tilde{R}^{\prime \prime}=\tilde{R}+P Q-(1 / 2) L$. The symmetry $U(1)_{\tilde{R}^{\prime \prime}}$ is an extension of $U(1)_{R^{\prime \prime}}$ in Eq.(6), contains $Z_{2}$ baryon parity and implies $U(1)_{B}$ to all orders.

Finally, it should be pointed out that $\nu^{c}$ and $T, \bar{T}$ can coexist with all the couplings mentioned being present. In this 'combined' case, $W$ possesses a $U(1)$ Peccei-Quinn (R-) symmetry, $U(1)_{\hat{P Q}}\left(U(1)_{\hat{R}}\right)$, which coincides with $U(1)_{P Q^{\prime}}\left(U(1)_{\tilde{R}^{\prime}}\right)$ or $U(1)_{P Q^{\prime \prime}}\left(U(1)_{\tilde{R}^{\prime \prime}}\right)$ when restricted to the superfields where these symmetries are defined. The R-symmetry $U(1)_{\hat{R}}$ implies $U(1)_{B}$ to all orders.

We have focused in this paper on MSSM and its extensions, with $Z_{2}$ matter parity embedded, to begin with, in a $U(1)$ R-symmetry. Non-zero neutrino masses, consistent with a redefined $U(1)$ R-symmetry, can be introduced in at least two ways. By requiring the higher order superpotential couplings to respect this redefined R-symmetry, one can i) explain proton stability to be a consequence of an automatic $U(1)_{B}$, and ii) show that the observed baryon asymmetry can arise via a primordial lepton asymmetry provided right handed neutrinos are present. Finally, simultaneous resolutions of the strong CP and $\mu$ problems, with $\mu \sim f_{a}^{2} / M_{P}$, can be elegantly accommodated in this scheme.

One of us (G. L.) would like to thank G. Dvali for drawing his attention to the resolution of the $\mu$ problem via a PQ-symmetry and for important discussions on this point. We acknowledge the NATO support, contract number NATO CRG-970149. One of us (Q.S.) would also like to acknowledge the DOE support under grant number DEFG02-91ER40626. 


\section{REFERENCES}

[1] G. Dvali, G. Lazarides and Q. Shafi, Phys. Lett. B424 (1998) 259.

[2] For another approach to MSSM with R-symmetry see L. J. Hall and L. Randall, Nucl. Phys. B352 (1991) 289.

[3] M. Gell-Mann, P. Ramond and R. Slansky, in Supergravity, Proceedings of the Workshop, Stony Brook, New York, 1979, eds. P. Van Nieuwenhuizen and D. Z. Freedman (North Holland, Amsterdam, 1979), p. 315; T. Yanagida, in Proceedings of the Workshop on Unified Theories and Baryon Number in the Universe, Tsukuba, Japan, 1979, eds. A. Sawada and A.Sugamoto (KEK Rep. No. 79-18, Tsukuba, Japan, 1979).

[4] C. Wetterich, Nucl. Phys. B187 (1981) 343; G. Lazarides, Q. Shafi and C. Wetterich, Nucl. Phys. B181 (1981) 287; R. N. Mohapatra and G. Senjanovic, Phys. Rev. D23 (1981) 165; R. Holman, G. Lazarides and Q. Shafi, Phys. Rev. D27 (1983) 995.

[5] E. Ma and U. Sarkar, hep-ph/9802445.

[6] M. Fukugita and T. Yanagida, Phys. Lett. B174 (1986) 45; W. Buchmüller and M. Plümacher, Phys. Lett. B389 (1996) 73. In the context of inflation see G. Lazarides and Q. Shafi, Phys. Lett. B258 (1991) 305; G. Lazarides, C. Panagiotakopoulos and Q. Shafi, Phys. Lett. B315 (1993) 325.

[7] G. Lazarides, R. Schaefer and Q. Shafi, Phys. Rev. D56 (1997) 1324; G. Lazarides, hep-ph/9802415 (to appear in the proceedings of the 6th BCSPIN Summer School).

[8] L. E. Ibáñez and F. Quevedo, Phys. Lett. B283 (1992) 261.

[9] R. Peccei and H. Quinn, Phys. Rev. Lett. 38 (1977) 1440; S. Weinberg, Phys. Rev. Lett. 40 (1978) 223; F. Wilczek, Phys. Rev. Lett. 40 (1978) 279.

[10] G. Lazarides, C. Panagiotakopoulos and Q. Shafi, Phys. Rev. Lett. 65 (1986) 432.

[11] For a recent discussion see H. P. Nilles and N. Polonsky, Nucl. Phys. B484 (1997) 33.

[12] J. E. Kim and H. P. Nilles, Phys. Lett. 138B (1984) 150.

[13] G. Dvali, private communication. 


\section{FIGURES}
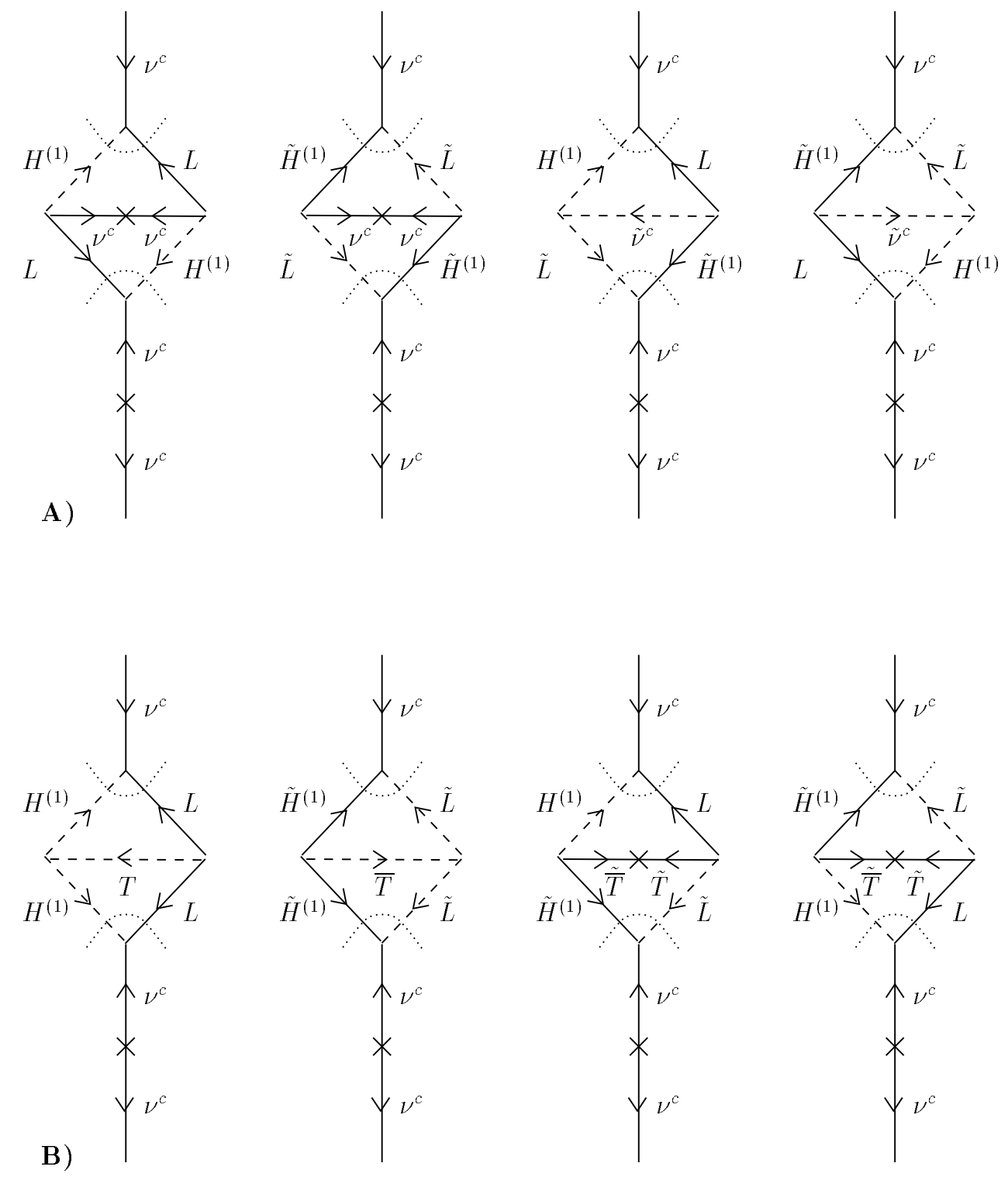

FIG. 1. The double-cut diagrams for leptogenesis via the decay of fermionic $\nu^{c}$ 's. The diagrams A (B) correspond to the exchange of $\nu^{c}(T, \bar{T})$ superfields. Continuous (dashed) lines represent fermions (bosons) while tildes denote the supersymmetric partners. 